Tecno Lógicas

ISSN 0123-7799

Vol. 18 , No. 35 , pp. 35-44

Julio-diciembre de 2015

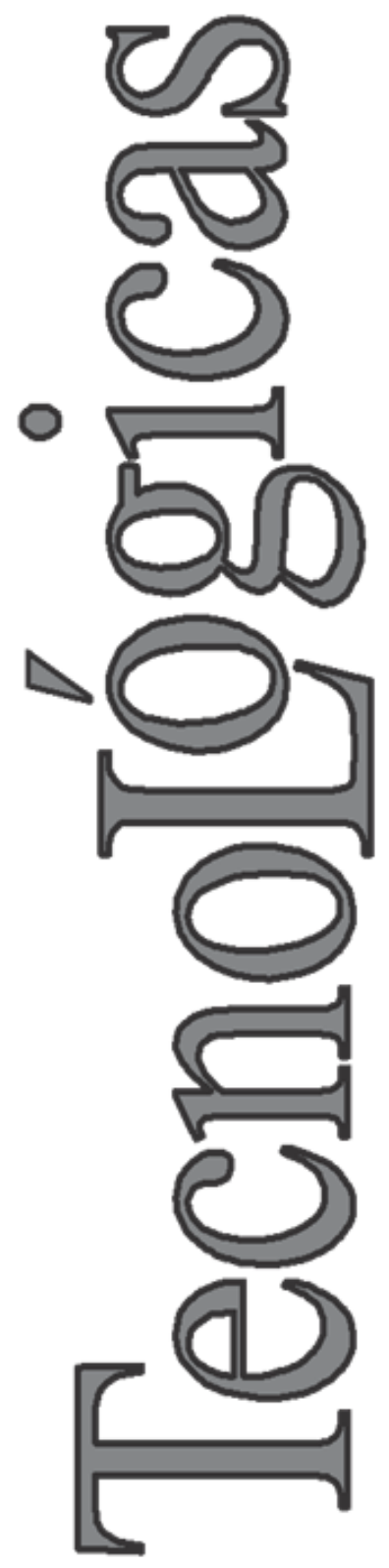

(C) Copyright 2015 por autores y Tecno Lógicas Este trabajo está licenciado bajo una Licencia Internacional Creative Commons Atribución (CC BY)

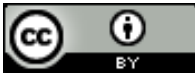

\section{Evaluación de un modelo estadístico para estimar la radiación solar en Magdalena, Colombia}

\section{Evaluation of a statistical model for estimating the solar radiation in Magdalena, Colombia}

\author{
Andrés M. Vélez-Pereira ${ }^{1}$, Eliana L. Vergara- \\ Vásquez ${ }^{2}$, William D. Barraza-Coronell ${ }^{3}$ \\ y Diana C. Agudelo-Yepes ${ }^{4}$
}

Recibido: 24 de agosto de 2014,

Aceptado: 30 de abril de 2015

Cómo citar / How to cite

A. M. Vélez-Pereira, E. L. Vergara-Vásquez, W. D. Barraza-Coronell y D. C. Agudelo-Yepes "Evaluación de un modelo estadístico para estimar la radiación solar en Magdalena, Colombia”, Tecno Lógicas, vol. 18, no. 35 , pp. 35-44, 2015.

1 M. Sc. Tecnología Ambiental, Grupo de Investigación Gestión y Sostenibilidad Ambiental, Universidad de la Costa, BarranquillaColombia, ing.andresvelez@gmail.com

2 M. Sc. Ciencias Ambientales, Programa de Ingeniería Ambiental, Facultad de Ciencias Ambientales, Universidad de la Costa, Barranquilla-Colombia, evergara6@cuc.edu.co

3 Estudiante de Ingeniería Ambiental, Programa de Ingeniería Ambiental, Facultad de Ciencias Ambientales, Universidad de la Costa, Barranquilla-Colombia, william.barraza15@gmail.com

4 Ingeniera ambiental, Programa de Ingeniería Ambiental, Facultad de Ciencias Ambientales, Universidad de la Costa, BarranquillaColombia, dagudelo11@gmail.com 


\section{Resumen}

La radiación solar global se establece como una variable meteorológica necesaria para en el entendimiento de los procesos bioquímicos del ambiente; su medición es compleja y requiere un alto costo de instalación, operación y mantenimiento. Su estimación se puede realizar mediante fórmulas empíricas basadas fundamentalmente en su geoposicionamiento, no obstante la precisión de los resultados obtenidos no es adecuada para el entendimiento de los procesos evaluados. El objetivo del presente trabajo fue establecer un modelo estadístico de correlación para estimar la radiación solar global a partir de los datos meteorológicos alternos. La información meteorológica fue suministrada por el Instituto de Hidrología, Meteorología y Estudios Ambientales de Colombia, que proporcionó la medición de radiación solar, temperatura, humedad relativa y horas de sol. Se plantearon regresiones simples entre las variables y la radiación solar; y regresiones múltiples entre las posibles combinaciones de las variables y la radiación solar. Se evidenció una buena correlación estadística entre las variables radiación solar, temperatura y humedad relativa, y poca relación con las horas de sol; siendo la humedad relativa la que guarda una mayor correlación y por tanto influencia en el modelo. Los modelos multivariados ( $\mathrm{R}^{2}$ promedio $\left.11,91 \%\right)$ presentaron mejor ajuste comparado con los modelos simples ( $\mathrm{R}^{2}$ promedio 5,66\%). El mejor modelo lo establece la regresión múltiple en la que se incluyen todas las variables ( $\mathrm{R}^{2}$ de $\left.13,75 \%\right)$, no siendo este el modelo más simple para su estimación.

\section{Palabras clave}

Radiación solar, estimación de la radiación solar, modelo estadístico de correlación.

\section{Abstract}

The global solar radiation is an important meteorological parameter for understanding the biochemical processes in the environment. Their measurement is complex and requires a high cost of installation, operation and maintenance, but this can be estimated by empirical equations based primarily on their geolocation. However, the accuracy of the results obtained is not suitable for understanding the processes evaluated. The aim of this study was to establish a statistical correlation model for estimating global solar radiation from alternative meteorological parameter. The meteorological information was provided by the Institute of Hydrology, Meteorology and Environmental Studies of Colombia, which provided the measurement of solar radiation, temperature, relative humidity and sunshine of the day for a year. Simple regressions between variables and solar radiation were raised; and multiple regressions between the possible combinations of each variables and solar radiation. The results show a good statistical correlation between variables solar radiation, temperature and relative humidity and little relation to the hours of sunshine, where relative humidity has the higher correlation and therefore is the parameter that more influence has on the model. The multivariate models ( $\mathrm{R}^{2}$ average $\left.11.91 \%\right)$ have better fit than simple models $\left(\mathrm{R}^{2}\right.$ average 5.66\%). The best model is multivariate regression in which all variables were included ( $\mathrm{R}^{2}$ of $\left.13.75 \%\right)$ although not the simplest model estimation.

\section{Keywords}

Solar radiation, solar radiation estimation, statistical correlation model. 


\section{INTRODUCCIÓN}

Los datos de radiación solar son de gran importancia por su aplicación en diversos campos; principalmente para el análisis de la carga térmica en edificios ubicados en zonas donde se presentan estaciones climáticas, optimizando el uso de aires acondicionados en las épocas de verano y disminuyendo el uso de equipos de calefacción en épocas de invierno, repercutiendo en ambos casos en una disminución de la demanda energética para satisfacer la necesidades [1], [2]. Adicionalmente los datos de la radiación solar se emplean en la planificación de las operaciones en plantas de energía renovables, meteorología, ciencias agrícolas, salud, implementación de nuevas tecnologías y también para algunos análisis de impacto ambiental [3].

Otro de los aspectos en lo que resulta importante la medición de la radiación solar, radica en el hecho que muchas de las actividades humanas que se realizan al aire libre, se asocian a enfermedades por exposición a los rayos ultravioleta de onda larga (UVA) y rayos ultravioleta de onda corta (UVB), soportados por el incremento de la radiación presentada desde los años setenta. Según lo señalado por algunas investigaciones médicas, la radiación ultravioleta ocasiona el fotoestrés oxidativo de la piel mediado por mecanismos tales como la absorción, reflexión o dispersión; sus consecuencias pueden ser varias: fenómenos inflamatorios, cáncer, tumores malignos, inmunosupresión, arrugas y envejecimiento acelerado, daños celulares y estructurales [4]. La exposición permanente a la radiación solar representa un riesgo para los intestinos, produce diarrea, nauseas, vómito, resequedad en los labios, obesidad, fallas en órganos y sistemas vitales, úlceras en la boca, artritis y afecta el crecimiento, entre muchos otros efectos nocivos [5], [6]. La incidencia del cáncer de piel continua en aumento, en el Reino Unido el cáncer de piel es el más común con 67000 casos de melanoma diagnosticados en 2002 [7]. Para el caso de Colombia se presenta gran desinformación en el tema de los efectos nocivos de la radiación solar debido a la inexistencia o deterioro de las estaciones meteorológicas para la medición de la radiación solar ultravioleta.

Además de la afección e influencia de la radiación solar para los seres humanos o la ventaja que representa en las construcciones y el uso como energía renovable; también presenta relevancia en los estudios realizados a los ecosistemas [8]. La interacción entre la radiación solar y los diferentes factores ambientales, forma parte de los procesos biológicos como la fotosíntesis, la absorción de nutrientes y la humedad del suelo para el equilibrio de los ecosistemas, funcionando como factores vitales para la productividad de los mismos [9].

La radiación solar no solo calienta la atmosfera, es también la causante de los desequilibrios de la química atmosférica, debido a la iniciación de procesos químicos y a la posterior contaminación por la dispersión y transporte en la dirección del viento predominante de los contaminantes producto del desequilibrio [10].

En la actualidad, existen muchos equipos destinados a medir y cuantificar la radiación solar global, dentro de los más importantes se encuentran los solarímetros o actinógrafos (heliógrafo), pirheliómetro, pirheliómetro de incidencia normal, fotómetro solar, piranómetro, piranómetro espectral, pirgeómetro y las estaciones meteorológicas [11]. Sin embargo, la disponibilidad de equipos que garanticen la información es escasa en muchos lugares, puesto que los costos de mantenimiento y calibración que requieren los equipos de medición son muy altos. Pese a estas limitaciones de la cobertura de medición, surge la posibilidad de emplear modelos de estimación de la radiación solar a partir de otros datos meteorológicos presentes en la zona de estudio [12].

En la literatura hay varios métodos empíricos utilizados para estimar la radiación solar global, en la que se expresa como 
función de otros parámetros como las horas de sol, nubosidad, entre otros parámetros meteorológicos [13]. No obstante, la utilización de modelos estadísticos está siendo empleada en los últimos años [14]-[16]; uno de los modelos iniciales es el de regresión de Angström. Investigaciones como las de [17]-[19] afirman que Angström propuso una relación lineal entre la proporción global de irradiación $\left(\mathrm{H} / \mathrm{H}_{0}\right)$ para la radiación global en días despejados y la proporción de las horas del sol ( $\left.\mathrm{S} / \mathrm{S}_{0}\right)$ para la duración astronómica del día. Los modelos paramétricos presentan una complejidad intermedia, que se basan en el conocimiento de variables atmosféricas frecuentemente disponibles o derivables a partir de otros datos, como el contenido de vapor de agua, espesor óptico de aerosoles y ozono, visibilidad, etc. [20]. Los modelos paramétricos no simulan las interacciones a escala de moléculas o partículas, sino que emplean distintos algoritmos para el cálculo de las transmitancias integrales de los diferentes componentes atmosféricos combinados en distintas formas de la ecuación de transporte, pudiendo representar de forma precisa los efectos de estos componentes sobre la radiación solar [21].

A nivel internacional se han desarrollado estudios y metodologías para establecer modelos de estimación de la radiación solar a partir de variables meteorológicas alternativas. Es así como empleando las ecuaciones previamente establecidas por Angström y otros, se han modelado la radiación solar diaria a partir de otros parámetros meteorológicos, algunos ejemplos lo presentan Bakirci [19] en Turquía, Jin et al. [22] and Wu et al. [23] en China, Hocaoğlu et al. [24] en Argelia y Okundamiya et al. [25] en Nigeria. Por otro lado, se han empleado métodos de regresiones robustos (Redes Neuronales Artificiales - ANN, Modelo autorregresivo integrado de media móvil - ARIMA, entre otros métodos) logrando establecer modelos de estimación de la radiación solar diaria, donde Almorox et al. [26] en España, Benghanem et al.
[27] en Arabia Saudita y Paoli et al. [28] en Francia son algunos de estos ejemplos. En el contexto nacional solo se cuenta con un primer acercamiento desarrollado en la ciudad de Bogotá [11], en el cual solo han obtenido los coeficientes de ecuaciones paramétricas establecidas y descritas por Yorukoglu y Celik [17].

Para el caso puntual de Colombia, la carencia de información homogénea y veraz de radiación solar es escasa, por lo que la presente propuesta busca establecer un primer acercamiento sobre los modelos de estimación de la radiación solar. El objetivo abordado es verificar la aplicación regresiones simples y multivariadas, a un conjunto de datos de meteorológicos proporcionados por el Instituto de Hidrología, Meteorología y Estudios Ambientales de Colombia (IDEAM), con el fin de establecer su posible utilización, en futuras estimaciones.

\section{METODOLOGÍA}

La información meteorológica empleada en el presente trabajo fue suministrada por el IDEAM, tomando como referencia la estación meteorológica 29065030 Prado Sevilla, localizada en el departamento del Magdalena, Colombia, en las coordenadas $10^{\circ} 45^{\prime}$ latitud norte y $74^{\circ} 09^{\prime}$ longitud oeste, a una altura de $18 \mathrm{msnm}$. Esta fue seleccionada por ser la estación meteorológica que cuenta con la medición de la radiación solar, temperatura, humedad y horas de sol cercanas. Los datos empleados corresponden al valor diario del periodo de tiempo comprendido entre marzo de 2011 y enero de 2012.

Una vez obtenida la información meteorológica se procedió a ordenar y procesarla con el fin de obtener las parejas de datos que serán alimentadas para la realización de las regresiones planteadas, esto teniendo en cuenta que la información suministrada no es continua y que en muchas ocasiones presenta datos faltantes. Primero, 
se realizó un análisis de varianza (ANOVA) tomando como factor de influencia las observaciones en un mismo mes con el fin de establecer el comportamiento general de las variables. Posteriormente, se desarrolló un análisis de correlación de Spearman entre las variables alternas y la radiación solar medida, lo que permite no sesgar el análisis por problemas de normalidad y linealidad ausentes; además de ser menos susceptible a los valores atípicos de lo esperado, disminuyendo así el error en el estadístico.

Finalmente, para la estimación del modelo estadístico que represente en mejor ajuste para los datos se establecieron tres diseños, teniendo en cuenta lo planteado por Benghanem et al. [27]. El primer grupo corresponde a regresiones simples entre cada una de las variables alternas y la radiación solar, el segundo grupo de diseño que se abordó lo establecen regresiones multivariadas entre un par de los parámetros alternos y la radiación solar. Finalmente, el tercer diseño lo conforma una regresión multivariada de todos los parámetros alternos y la radiación solar medida. En general se obtuvieron un total de siete regresiones (Tabla 1 ). El modelo de regresión que presente la mejor predicción será aquel que presente estadísticamente un mejor coeficiente de correlación $\left(\mathrm{R}^{2}\right)$ y un menor Error Absoluto Medio (MAE).

Tabla 1. Descripción de las funciones empleadas en las regresiones simples y multivariadas. Fuente: Autores

\begin{tabular}{lll}
\hline $\begin{array}{c}\text { Tipo de } \\
\text { regresión }\end{array}$ & \multicolumn{1}{c}{ Función } & \multicolumn{1}{c}{$\begin{array}{c}\text { Significado de las } \\
\text { variables }\end{array}$} \\
\hline Simple & $f\{t, H\}$ & $\begin{array}{l}\text { Temperatura (t), } \\
\text { Radiación solar (H) } \\
\text { Humedad relativa (HR), } \\
\text { Radiación solar (H) } \\
\text { Horas de sol (S), } \\
\text { Radiación solar }(\mathrm{H})\end{array}$ \\
\hline $\begin{array}{l}\text { Combinadas } \\
\text { dos }\end{array}$ & $f\{S, H\}$ & \\
\hline $\begin{array}{l}\text { Combinadas) } \\
\text { general }\end{array}$ & $f\{H R, S ; H\}$ & \\
\hline
\end{tabular}

\section{RESULTADOS Y DISCUSIÓN}

Los datos muestran que las horas de sol fluctúan en un rango no muy amplio de duración comprendidos entre 11,45 y 12,65 horas, así mismo se observa que los meses centrales del año presentan una mayor duración de las horas de sol (ver Fig. 1a). Igualmente, los datos presentan una baja dispersión mensual (datos recolectados del mismo mes), esto se puede atribuir al hecho de que la zona de estudio se encuentra en una zona cercana al ecuador y de poca altitud, presentando poca variación estacional de las horas del sol a lo largo del mes o el año. En cuanto al ANOVA de las horas de sol, se ratifica lo planteado anteriormente, puesto que se observa la mayor conformación de grupos homogéneos de todos los parámetros estudiados (Tabla 2), debido a la poca variación intrínseca de los datos en un mismo mes.

Para el caso de la humedad relativa los datos dentro de un mismo mes reportan una mayor variabilidad comparada con la duración de las horas de sol, presentando un ligero descenso de la humedad los primeros meses del año (ver Fig. 1b). No obstante, la humedad relativa para esta zona se reportó por encima del 72\%. Así mismo, los datos de humedad relativa se ajustan a una distribución normal en los valores recolectados a lo largo del año de muestreo. Esta amplia variación intermensual es lo que permite que se obtenga una cantidad de grupo homogéneos en la prueba ANOVA (Tabla 2), manifestando que a lo largo del año existe una variación significativa de la humedad relativa.

La temperatura reportada para el año de muestro evidencia según la Fig. 1c una amplia variabilidad intermensual, no obstante esta variación a lo largo del año es escasa o muy estrecha variando entre 23,9 y $30,5{ }^{\circ} \mathrm{C}$. Sin embargo, el ANOVA reporta una misma cantidad de grupos homogéneos (Tabla 2) que la humedad relativa, permitiendo inferir que existe una variación anual de la temperatura, pese a estar 
ubicada en una zona ecuatorial. Por último, los valores de radiación solar presentan alta variabilidad los meses de marzo y abril, los cuales van disminuyendo a medida que avanza el año hasta presentar una baja variabilidad (ver Fig. 1d). Esta información se corrobora en el ANOVA, donde se presenta la menor cantidad de grupos homogéneos en comparación con los parámetros restantes. No obstante, el ANOVA manifiesta al igual que en los casos anteriores la existencia de una variación anual en la radiación solar (Tabla 2 ).

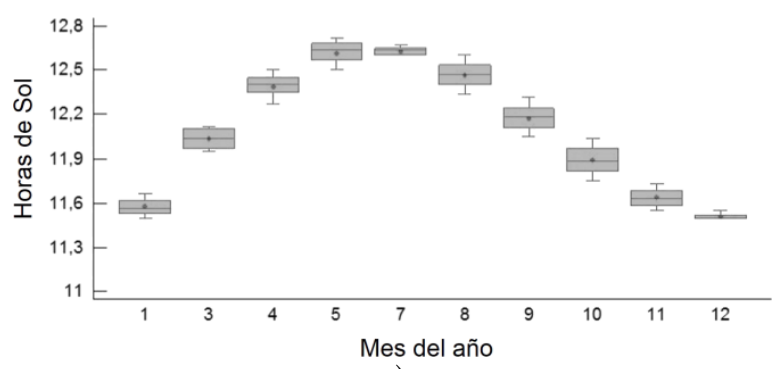

a)

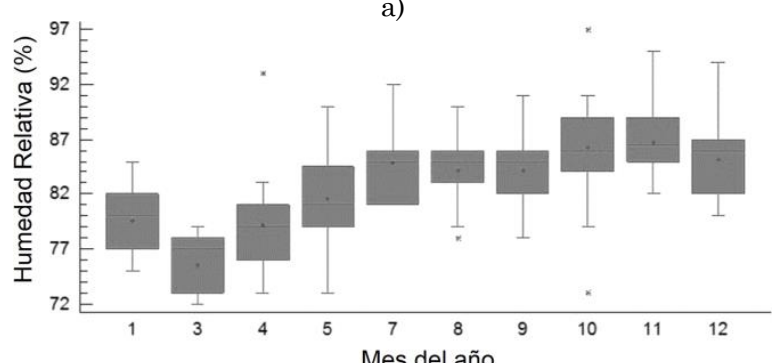

b)

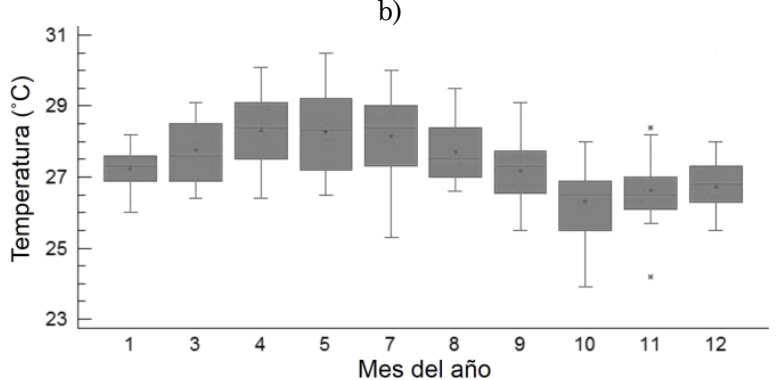

c)

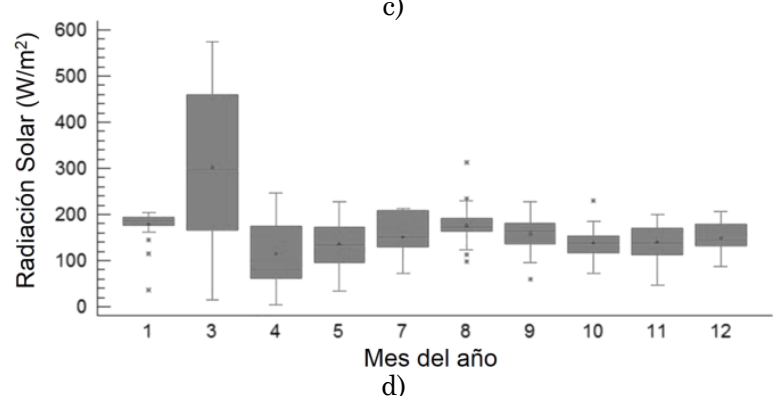

Fig. 1. Medias mensuales a lo largo de un año de las variables: a) Horas de sol, b) Humedad relativa, c) Temperatura, d) Radiación solar. Fuente: Autores
Tabla 2. Resumen de los análisis de las series de tiempo de las variables meteorológicas. Fuente: Autores

\begin{tabular}{ccccc}
\hline Parámetro & Rango & $\begin{array}{c}\text { Media } \\
\text { anual }\end{array}$ & $\begin{array}{c}\text { P-Valor } \\
\text { ANOVA }^{\text {a }}\end{array}$ & $\begin{array}{c}\text { Grupos } \\
\text { homogéneos }\end{array}$ \\
\hline S & $11,5-12,7$ & 12,04 & 0,00 & 9 \\
HR & $72,0-97,0$ & 83,33 & 0,00 & 5 \\
T & $23,9-30,5$ & 27,30 & 0,00 & 5 \\
H & $4,3-574,38$ & 154,0 & 0,00 & 4 \\
\hline
\end{tabular}

a P-crítico $(0,05)$, si P-valor < P-Crítico existe una diferencia estadísticamente significativa entre la media del parámetro entre un nivel de mes y otro, con un nivel del $95 \%$ de confianza. ${ }^{\mathrm{b}}$ Establecidos por el procedimiento de diferencia mínima significativa (LSD) de Fisher.

En general, el comportamiento de los datos meteorológicos muestran que a pesar de estar ubicados en cercanías al Ecuador, ser una zona de baja altitud y lejana de cuerpos de agua, presenta una variación de las medias mensuales a lo largo del año en las condiciones meteorológicas que inclusive para el caso de la temperatura y la humedad relativa se observa que puede llegar a ser mucho más variable al observarse la distribución de los registros de un mismo mes.

En cuanto al análisis de Spearman los resultados arrojaron que existe una fuerte correlación entre la temperatura $(0,309)$ y la humedad relativa $(-0,421)$ con un nivel de significancia $\mathrm{P}<0,01$. Mientras que las horas de sol no mostró una correlación significativa. Por otro lado, el análisis de Spearman permite establecer que las horas de sol y la temperatura tienen un efecto positivo sobre la radiación solar, mientras que la humedad relativa se presume de un efecto negativo. Teniendo en cuenta que las variables meteorológicas pueden estar relacionadas, se realizó un análisis de multicolinealidad entre las variables alternas mediante el factor de inflación de la varianza (VIF), para descartar la existencia de una relación o dependencia lineal entre los estimadores de la regresión (parámetros meteorológicos alternos). Se obtuvo un promedio de 1,88, el cual está por debajo del valor crítico 10 , descartando así la presencia de multicolinealidad. 
Para el caso del primer diseño de las correlaciones simples, en la Fig. 2a se observa la correlación presentada entre la radiación solar medida y la estimada por el modelo estadístico de correlación obtenido con la temperatura. Se presenta una relación directa, según lo presentado por el coeficiente $\mathrm{R}(0,21)$; no obstante este mismo coeficiente nos permite inferir una baja correlación de los pares de datos con un nivel de significancia del 95\%, corroborada con el coeficiente $\mathrm{R}$ cuadrado $\left(\mathrm{R}^{2}\right)$, que presenta un coeficiente de $4,51 \%$.

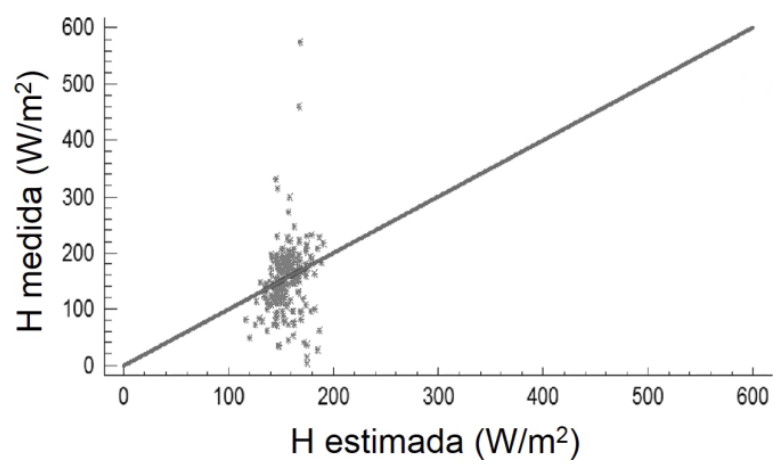

a)

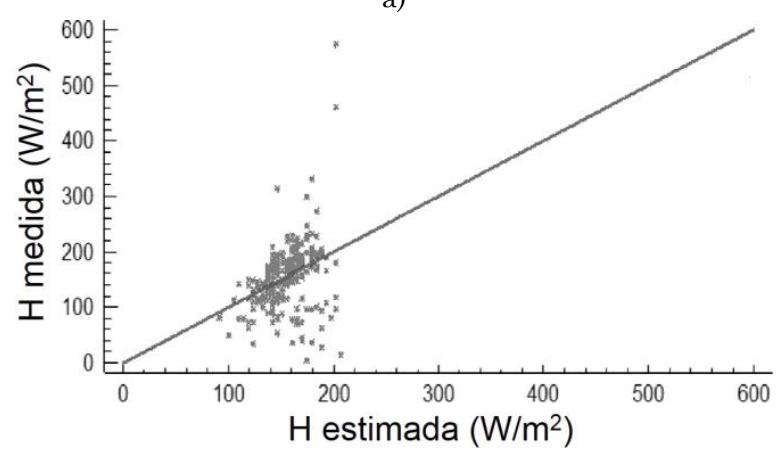

b)

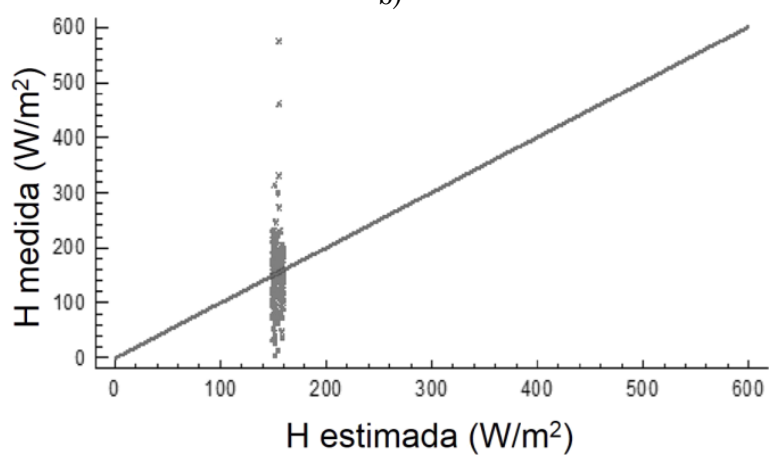

c)

Fig. 2. Comparación entre la radiación solar medida y estimada mediante el modelo establecido con a) la temperatura, b) la humedad relativa, c) las horas de sol.

Fuente: Autores
Para el caso del modelo estadístico entre la radiación solar y la humedad relativa el coeficiente $\mathrm{R}(-0,35)$ indica una relación inversa entre las variables, ratificando lo presentado por el análisis de Spearman. El coeficiente de correlación $\mathrm{R}^{2}$ muestra una mayor relación en los datos en comparación con las demás regresiones aplicadas (ver Fig. 2b).

Por último, la última Fig. 2c presenta la correlación entre la radiación solar medida y la estimada mediante el modelo estadístico con la duración de las horas de sol, en esta se presenta una relación inversa según el coeficiente $\mathrm{R}(-0,06)$ y un coeficiente $\mathrm{R}^{2} \quad 0,36 \%$, presentando los valores más bajos de correlación; de este último modelo se esperaban mayores valores en los coeficientes debido a la relación directa entre las variables. No obstante, la baja relación se puede atribuir al hecho de la poca continuidad de recolección de los datos. En la Tabla 3 se resumen los resultados del análisis estadístico de las regresiones simples, así como también las ecuaciones que describen el mejor ajuste.

Los resultados obtenidos en cuanto a los coeficientes $\mathrm{R}^{2}$ en el presente estudio tienen amplia diferencia con respecto a los presentados por otros autores [13], [26], [27], [29], los cuales presentan para el caso de regresiones con temperatura un coeficiente cercano al $85 \%$. No obstante, los resultado de las presentes regresiones son mayores a los aplicados a las regresiones estadísticas preestablecidas por otros autores y aplicadas al mismo conjunto de datos, $\mathrm{R}^{2}$ para $\mathrm{S} 1,9 \%$; $\mathrm{R}^{2}$ para t $4,4 \% ; \mathrm{R}^{2}$ para $\mathrm{RH}$ $11,14 \%$ [30].

Para el diseño del segundo y tercer grupo, los modelos estadísticos ejecutados para los datos corresponden a las regresiones múltiples (ver Fig. 3), en estas se observa que la mayoría de ellas presentan correlación entre la radiación solar medida y estimada por los modelos, evidenciado por el aumento de los coeficientes $\mathrm{R}^{2}$, exceptuando a la función $f\{t, S, H\}$ que presenta un valor por debajo de $10 \%$. 


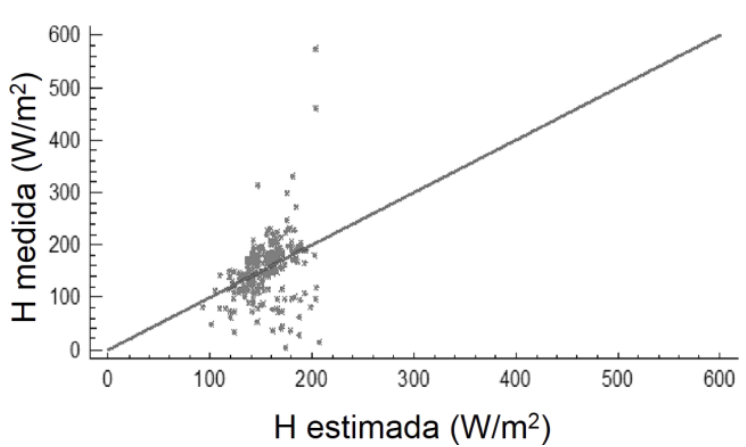

a)

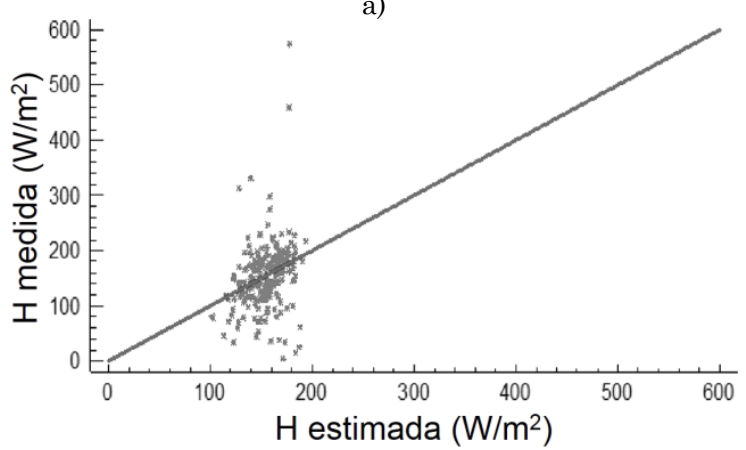

b)

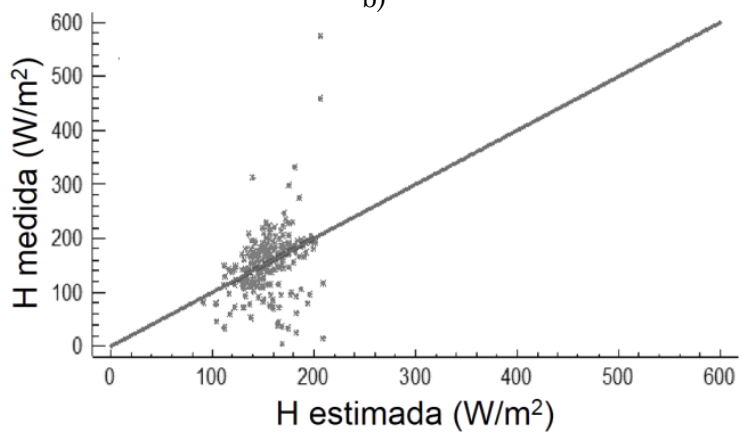

c)

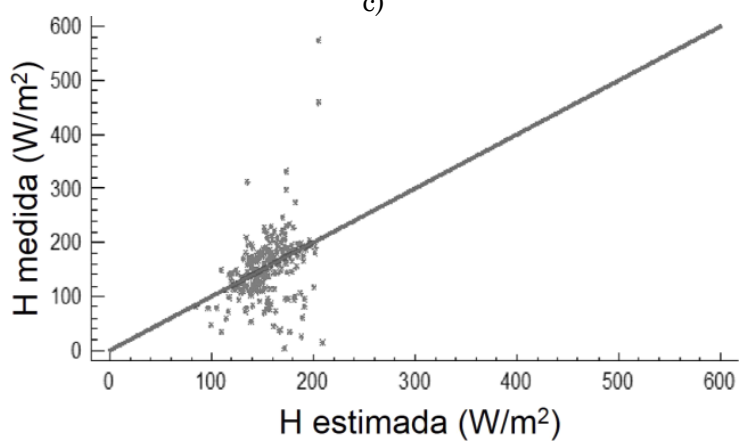

d)

Fig. 3. Comparación entre la radiación solar medida y la radiación solar estimada mediante el modelo establecido con: (a) temperatura y humedad relativa, (b) temperatura y horas de sol, (c) humedad relativa y horas de sol, (d) temperatura, humedad relativa y horas de sol. Fuente: Autores

Así mismo, en los diferentes modelos estadísticos aplicados, el análisis ANOVA sugiere que el parámetro de temperatura puede ser suprimido en las ecuaciones. En la Tabla 4 se resumen los parámetros esta- dísticos de las regresiones. $\mathrm{Al}$ comparar los resultados con los presentados por otras investigaciones en las que se emplean parámetros meteorológicos alternos como variables de entrada en las regresiones [27], [31], [32], los valores del presente trabajo se encuentran por debajo de los presentados, los cuales varían entre $95 \mathrm{y}$ 99\% en el coeficiente $\mathrm{R}^{2}$. Sin embargo, los valores presentados de $\mathrm{R}^{2}$ en las regresiones múltiples son más altos que las regresiones de una sola variable, esto se puede atribuir al hecho que las condiciones de medición de la radiación solar, se pueden ver influenciadas por los entornos meteorológicos del área de estudio.

\section{CONCLUSIONES}

Las variables meteorológicas reportadas para el proyecto presentan poca variación en los datos observados en el mismo mes, no obstante reportan amplios cambios a lo largo del año. En cuanto a las correlaciones estadísticas, se puede concluir que los datos recopilados y observados no presentan un ajuste significativamente estadístico en los diferentes tipos de modelos estudiados. Sin embargo, sí se puede establecer que la humedad relativa guarda una mayor relación o ajuste en los modelos con la radiación solar permitiendo concluir que es la variable climatológica que más influencia tiene sobre el modelo.

El modelo que presenta el mejor ajuste para predecir la radiación solar para el área de estudio es la regresión múltiple en la que se incluyen todas las variables, no siendo este el modelo más simple para su estimación. Se sugiere realizar los mismos procedimientos aquí presentados con datos multianuales, que permitan identificar con mayor precisión las variaciones multianuales a que tengan lugar las variables meteorológicas; pudiendo establecer una replicabilidad posible de los puntos, que permitan comprobar la influencia de cada uno en las regresiones planteadas. 
Evaluación de un modelo estadístico para estimar la radiación solar en Magdalena, Colombia

Tabla 3. Resumen de los parámetros estadísticos determinados en los modelos de regresión simple. Fuente: Autores

\begin{tabular}{cccccc}
\hline \multirow{2}{*}{ Función } & \multicolumn{2}{c}{ Coef. de la ecuación } & Coef. $\mathrm{R}^{2}$ & $\begin{array}{c}\text { Error Absoluto Medio } \\
\text { (MAE) }\end{array}$ & $\begin{array}{c}\text { Ecuación del mejor } \\
\text { ajuste }\end{array}$ \\
\cline { 2 - 3 } $\boldsymbol{f}\{\boldsymbol{t}, \boldsymbol{H}\}$ & $-152,21$ & 11,24 & $4,51 \%$ & 37,78 & $\boldsymbol{H}=\boldsymbol{\beta}_{\mathbf{1}}+\boldsymbol{\beta}_{\mathbf{2}} \frac{\mathbf{1}}{\boldsymbol{t}}$ \\
$\boldsymbol{f}\{\boldsymbol{H} \boldsymbol{R}, \boldsymbol{H}\}$ & 541,24 & $-4,64$ & $12,11 \%$ & 35,60 & $\boldsymbol{H}=\sqrt[2]{\boldsymbol{\beta}_{\mathbf{1}}+\boldsymbol{\beta}_{2} \frac{\mathbf{1}}{\boldsymbol{H} \boldsymbol{R}}}$ \\
$\boldsymbol{f}\{\boldsymbol{S}, \boldsymbol{H}\}$ & 256,24 & $-8,45$ & $0,36 \%$ & 41,14 & $\boldsymbol{H}=\boldsymbol{e}^{\left(\boldsymbol{\beta}_{1}+\boldsymbol{\beta}_{2} s^{2}\right)}$ \\
\hline
\end{tabular}

Coef. $=$ Coeficiente, $b_{1}=$ Término independiente, $b_{2}=$ Coeficiente de la variable

Tabla 4. Resumen de las regresiones lineales múltiples. Fuente: Autores

\begin{tabular}{|c|c|c|c|c|c|c|}
\hline \multirow{2}{*}{ Función } & \multicolumn{4}{|c|}{ Coeficientes de la ecuación } & \multirow{2}{*}{ Coef. $\mathrm{R}^{2}$} & \multirow{2}{*}{$\begin{array}{l}\text { Error Absoluto } \\
\text { Medio (MAE) }\end{array}$} \\
\hline & $B_{1}$ & $B_{2}$ & $B_{3}$ & $B_{4}$ & & \\
\hline$f\{t, H R ; H\}$ & $0,83^{*}$ & $-4,77$ & -- & 574,98 & $12,13 \%$ & 35,68 \\
\hline $\boldsymbol{f}\{\boldsymbol{t}, \boldsymbol{S} ; \boldsymbol{H}\}$ & 17,39 & -- & $-32,26$ & 68,11 & $8,34 \%$ & 36,41 \\
\hline$f\{S, H R ; H\}$ & -- & $-4,87$ & $-16,25$ & 756,18 & $13,40 \%$ & 35,34 \\
\hline $\boldsymbol{f}\{\boldsymbol{t}, \boldsymbol{H R}, \boldsymbol{S} ; \boldsymbol{H}\}$ & 4,85 & $-4,17$ & $-21,77$ & 631,83 & $13,75 \%$ & 34,80 \\
\hline
\end{tabular}

Coef. = Coeficiente, $B_{1}=$ Coeficiente de temperatura, $B_{2}=$ Coeficiente de humedad relativa

$B_{3}=$ Coeficiente de horas de sol, $B_{4}=$ Término independiente

\section{AGRADECIMIENTOS}

Los autores expresan sus agradecimientos a la Universidad de la Costa por el apoyo financiero para el desarrollo del proyecto de investigación.

\section{REFERENCIAS}

[1] A. Hematian, Y. Ajabshirchi, H. Behfar, and H. Ghahramani, "Designing, construction and analysis of speed control system of the fan with PV feeding source in an air solar collector," Mod. Appl. Sci., vol. 6, no. 1, pp. 136-144, 2012.

[2] A. Fayadah, "Experimental Investigation of Thermal Performance of Solar Cooker with Reflector,” Eur. J. Sci. Res., vol. 56, no. 1, p. 112, 2011.

[3] A. Mellit and A. M. Pavan, "A 24-h forecast of solar irradiance using artificial neural network: Application for performance prediction of a gridconnected PV plant at Trieste, Italy," Sol. Energy, vol. 84, no. 5, pp. 807-821, May 2010.

[4] E. D. de Villegas, M. D. C. Menéndez, M. S. Martínez, M. C. Alonso, and N. R. Viera, "Radiación ultravioleta. Fotoenvejecimiento cutáneo.," Rev. Electrónica las Ciencias Médicas en Cienfuegos, vol. 3, no. 1, pp. 14-33, 2005.

[5] S. Magee, Solar Radiation, Global Warming and Human Disease. Steven Magee, 2010.
[6] P. E. Stoebner, M. Rahmoun, C. Ferrand, L. Meunier, H. Yssel, and J. Pene, "A single suberythematous exposure of solar-simulated radiation on the elicitation phase of contact hypersensitivity induces IL-10-producing Tregulatory cells in human skin," Exp. Dermatol., vol. 15, no. 8, pp. 615-624, Aug. 2006.

[7] R. Haywood, C. Andrady, N. Kassouf, and N. Sheppard, "Intensity-dependent Direct Solar Radiation- and UVA-induced Radical Damage to Human Skin and DNA, Lipids and Proteins," Photochem. Photobiol., vol. 87, no. 1, pp. 117130, Jan. 2011.

[8] J. Almorox, M. Benito, and C. Hontoria, "Estimation of global solar radiation in Venezuela," Interciencia, vol. 33, no. 4, pp. 280283, 2008.

[9] P. C. Cavatte, J. B. Zonta, J. C. Lopes, L. T. de Souza, J. H. Zonta, and R. P. Cavatte, "Germinação e vigor de sementes de cenoura em solo de mineração de calcário sob diferentes intensidades luminosas e adubações," Idesia (Arica), vol. 27, no. 2, pp. 25-32, 2009.

[10] G. C. Rojas, C. M. Rodríguez, S. C. Cervantes, and M. Morán-Pineda, "Contaminación del aire urbano: efectos de la radiación solar," in XXVIII Congreso Interamericano de Ingeniería Sanitaria y Ambiental, 2002.

[11] I. Mendoza and D. Piedra, "Validación de modelos de radiacion solar directa para la ciudad de Bogotá a partir de datos experimentales," Rev. la Soc. Colomb. Física, vol. 38, no. 4, pp. 1435-1438, 2006. 
[12] L. T. Wong and W. K. Chow, "Solar radiation model,” Appl. Energy, vol. 69, no. 3, pp. 191-224, Jul. 2001.

[13]J. Almorox, M. Bocco, and E. Willington, "Estimation of daily global solar radiation from measured temperatures at Cañada de Luque, Córdoba, Argentina," Renew. Energy, vol. 60, pp. 382-387, 2013.

[14] F. Ahwide, A. Spena, and A. El-Kafrawy, "Correlation for the Average Daily Diffuse Fraction with Clearness Index and Estimation of Beam Solar Radiation and Possible Sunshine Hours Fraction in Sabha, Ghdames and Tripoli Libya," APCBEE Procedia, vol. 5, pp. 208-220, 2013.

[15] S. Bhardwaj, V. Sharma, S. Srivastava, O. S. Sastry, B. Bandyopadhyay, S. S. Chandel, and J. R. P. Gupta, "Estimation of solar radiation using a combination of Hidden Markov Model and generalized Fuzzy model," Sol. Energy, vol. 93, pp. 43-54, 2013.

[16] H. Khorasanizadeh and K. Mohammadi, "Prediction of daily global solar radiation by day of the year in four cities located in the sunny regions of Iran," Energy Convers. Manag., vol. 76, pp. 385-392, Dec. 2013.

[17] M. Yorukoglu and A. N. Celik, "A critical review on the estimation of daily global solar radiation from sunshine duration," Energy Convers. Manag., vol. 47, no. 15-16, pp. 2441-2450, Sep. 2006.

[18] I. T. Toğrul, "Estimation of solar radiation from angstroms coefficients by using geographical and meteorological data in Bishkek, Kyrgyzstan., J. Therm. Sci. Technol., vol. 29, no. 2, pp. 99-108, 2009.

[19] K. Bakirci, "Correlations for estimation of daily global solar radiation with hours of bright sunshine in Turkey," Energy, vol. 34, no. 4, pp. 485-501, 2009.

[20] M. Fabre Morales, "Comparación de modelos de cielo despejado para la predicción a corto plazo de la irradiancia directa normal," Universidad de Salamanca, 2013.

[21] M. J. Lucano, F. M, and I. van E, "Evaluation of the global solar radiation potential in the department of Cochabamba (Bolivia) using models of geographic information systems and satellite images," Rev. Boliv. Física, vol. 17, no. 1562-3823, pp. 13-21, 2010.

[22]Z. Jin, W. Yezheng, and Y. Gang, "General formula for estimation of monthly average daily global solar radiation in China," Energy Convers. Manag., vol. 46, no. 2, pp. 257-268, Jan. 2005.

[23] G. Wu, Y. Liu, and T. Wang, "Methods and strategy for modeling daily global solar radiation with measured meteorological data - A case study in Nanchang station, China," Energy Convers. Manag., vol. 48, no. 9, pp. 2447-2452, Sep. 2007.

[24] F. O. Hocaoğlu, Ö. N. Gerek, and M. Kurban, "Hourly solar radiation forecasting using optimal coefficient 2-D linear filters and feedforward neural networks," Sol. Energy, vol. 82, no. 8, pp. 714-726, Aug. 2008.

[25] M. S. Okundamiya, J. O. Emagbetere, and E. A. Ogujor, "Evaluation of Various Global Solar Radiation Models for Nigeria," Int. J. Green Energy, vol. 0, no. ja, p. 150106045846008 , Jan. 2015.

[26] J. Almorox, C. Hontoria, and M. Benito, "Models for obtaining daily global solar radiation with measured air temperature data in Madrid (Spain)," Appl. Energy, vol. 88, no. 5, pp. 17031709, May 2011.

[27] M. Benghanem, A. Mellit, and S. N. Alamri, "ANN-based modelling and estimation of daily global solar radiation data: A case study," Energy Convers. Manag., vol. 50, no. 7, pp. 16441655, 2009.

[28] C. Paoli, C. Voyant, M. Muselli, and M.-L. Nivet, "Forecasting of preprocessed daily solar radiation time series using neural networks," Sol. Energy, vol. 84, no. 12, pp. 2146-2160, Dec. 2010.

[29]A. Rahimikhoob, "Estimating global solar radiation using artificial neural network and air temperature data in a semi-arid environment," Renew. Energy, vol. 35, no. 9, pp. 2131-2135, Sep. 2010.

[30]A. M. Vélez-Pereira, E. V. Vásquez, W. B. Coronell, and D. A. Yepes, "Determinación de un modelo paramétrico para estimar la radiación solar," Ingenium, vol. 7, no. 18, pp. 11-17, 2013.

[31]A. Azadeh, A. Maghsoudi, and S. Sohrabkhani, "An integrated artificial neural networks approach for predicting global radiation," Energy Convers. Manag., vol. 50, no. 6, pp. 1497-1505, 2009.

[32] M. A. Behrang, E. Assareh, A. Ghanbarzadeh, and A. R. Noghrehabadi, "The potential of different artificial neural network (ANN) techniques in daily global solar radiation modeling based on meteorological data," Solar Energy, vol. 84, no. 8. pp. 1468-1480, 2010. 